\title{
Smart Calcium Phosphate Bioceramic Scaffold for Bone Tissue
}

\section{Engineering}

\section{Guy Daculsi ${ }^{1, a}$, Thomas Miramond1 ${ }^{1,2, b}$, Pascal Borget ${ }^{2, c}$, Serge Baroth ${ }^{2, d}$}

\author{
${ }^{1}$ INSERM U791, LIOAD, Faculté de Chirurgie Dentaire Nantes France \\ ${ }^{2}$ Biomatlante, ZA des 4 Nations, Vigneux de Bretagne France \\ aguy.daculsi@univ-nantes.fr; bthomas.miramond@etu.univ-nantes.fr, \\ cpascalborget@biomatlante.com; d sergebaroth@biomatlante.com
}

Keywords: biphasic calcium phosphate, microporous, macroporous, scaffold, tissue engineering.

\begin{abstract}
The development of $\mathrm{CaP}$ ceramics involved a better control of the process of resorption and bone substitution. Micro Macroporous Biphasic $\mathrm{CaP},(\mathrm{MBCP}+\mathrm{TM})$ is a concept based on an optimum balance of the more stable phase of HA and more soluble TCP. The material is soluble and gradually dissolves in the body, seeding new bone formation as it releases $\mathrm{Ca}$ and $\mathrm{P}$ ions into the biological medium. The MBCP+ is selected for tissue engineering in a large European research program on osteoinduction and mesenchymal stem cell technology (REBORNE $7^{\text {th }}$ EU frame work program, Regenerative Bone defects using New biomedical Engineering approaches, www.reborne.org). We have optimized the matrices in terms of their physical, chemical, and crystal properties, to improve cell colonization and to increase kinetic bone ingrowth. The fast cell colonization and resorption of the material are associated to the interconnected macropores structure which enhances the resorption bone substitution process. The micropore content involves biological fluid diffusion and suitable adsorption surfaces for circulating growth factors. The bioceramics developed for this project was fully characterized using X-Ray diffraction, FTIR, X-rays micro tomography, Hg porosimetry, BET specific surface area, compressive mechanical test, and SEM. Preclinical tests on the optimized scaffold were realized in critical size defects in several sites of implantation and animals (rats, rabbits, goats, dogs). The smart scaffold has a total porosity of $73 \%$, constituted of macropores $(>100 \mu \mathrm{m})$, mesopores of 10 to $100 \mu \mathrm{m}$ and high micropores $(<10 \mu \mathrm{m})$ content of more or less $40 \%$. The crystal size is $<0.5$ to $1 \mu \mathrm{m}$ and the specific surface area was around $6 \mathrm{~m}^{2} / \mathrm{g}$.

The in vivo experiment indicated higher colonization by osteogenic cells demonstrating suitable matrices for tissue engineering. The HA/TCP ratio of 20/80 was also more efficient for combination with total bone marrow or stem cell cultivation and expansion before to be implanted.
\end{abstract}

\section{Introduction}

There are numerous evidences that the intrinsic MSC capacity to activate endogenous regenerative mechanisms and to recruit host cells in an ectopic bone formation model is critically dependent on MSC technology but also the physico chemical and structural properties of the scaffolds [1,2]. Nonetheless, for the success of the MSC transplant, the nature and the structure of scaffolds onto which cell are seeded is very critical. Since, in addition to represent a three dimensional carrier for the cells and a matrix with the correct shape within which the new tissue is formed, scaffolds are crucial in determining the optimal microenvironment for the cell to fully express their regenerative potential. A large Collaborative Project of the seventh framework program (topic HEALTH-2009-1.4.2 on Regenerative Bone defects using New biomedical Engineering approaches, REBORNE, P. Layrolle coordinator) is in progress to explore these scientific and clinical fields. The main objective of this proposal is to develop new biomaterials that stimulate bone tissue formation in combination with adult stem cells for regenerating bone defects in orthopaedic and maxillofacial surgery. 
We have optimized the new scaffolds based on a Micro Macroporous concept of Biphasic Calcium Phosphate (MBCP) with a micro structure highly suitable for tissue engineering techniques and showing improved osteogenic properties. In different animal models, a mixture of MBCP granules and total bone marrow appeared to be the most efficient among all the materials tested for bone substitution in difficult situation like irradiated areas, radionecrosis $[3,4]$.

The development of $\mathrm{CaP}$ ceramics for bone graft involves a better control of the process of biomaterials resorption and bone substitution. Bone graft materials biomaterials are largely represented by calcium phosphate hydroxyapatite $\mathrm{HA}$, tricalcium phosphate $\mathrm{TCP}$, and biphasic $\mathrm{CaP}$, MBCP. The concept based on biphasic CaP ceramics is achieved by an optimum balance of the more stable phase of HA and more soluble TCP. The material is soluble and gradually dissolves in the body, seeding new bone formation as it releases $\mathrm{Ca}$ and $\mathrm{P}$ ions into the biological medium [5]. These bioceramics are largely used for bone reconstruction and will be specially optimized for combination marrow during surgery or for bone tissue engineering using stem cells. We have optimized matrices in terms of their physico-chemical and crystal properties, to improve cell colonization and to increase the kinetic of bone ingrowth The fast cell colonization and resorption of the material are associated to the interconnected macropore structure which enhances the resorption bone substitution process. The micropore content involves biological fluids diffusion and suitable absorption surfaces for circulating growth factors. The objectives of this paper was to present the scientific scaffold approach in the REBORNE European project and why the MBCP+ bioceramics has been selected for the project on stem cells technology and clinical trials.

\section{Materials and methods}

Interconnected Micro Macroporous Biphasic $\mathrm{CaP}$ ceramic (MBCP+, $\mathrm{CE}$ mark and FDA 510k, Biomatlante SA France) was an improvement of the technology of microporous and macroporous calcium phosphate bioceramic developed twenty years ago [5]. Shortly, CaP deficient apatite CDA, were associated to a mixture of selected particles of porogen. After isostatic compaction, the block was sintered according a specific process of sublimation/calcinations at low temperature. The low sintering temperature presents the advantage to preserve high micropore content and smaller crystal than higher sintering temperature [6]. The obtained bioceramics was characterized using X-Rays, FTIR, X-rays microtomography, permeability, $\mathrm{Hg}$ porosimetry, BET specific surface area, mechanical testings, and SEM. Crystal structure at the nanoscale was observed and analyzed using high resolution transmission electron microscopy (hrTEM) and electronic diffraction (ED). The granules of $\mathrm{MBCP}+$ has been tested in several pre-clinical studies in goats, rabbits, rats, with or without cell addition (total bone marrow, mesanchymal stem cells) in critical size defects of femoral epiphysis, and non bony site to test osteogenicity/osteoinductive properties. The retrieved implants were analyzed by micro CT, light microscopy, SEM, and image analysis for histomorphometry and numerous publications were realized on biocompatibility, efficacy, performance, in animals models and in human clinical applications [7].

\section{Results}

XRays diffraction only showed Hydroxyapatite and $\beta$-TCP content. The HA/TCP ratio measured was 20/80 (figure 1). No trace of other calcium phosphate species could be observed.

FTIR confirms high purity of HA and TCP without any carbonate (figure 2).

Figure 1: $M B C P+X R D$ Figure 2: $M B C P+F T I R$
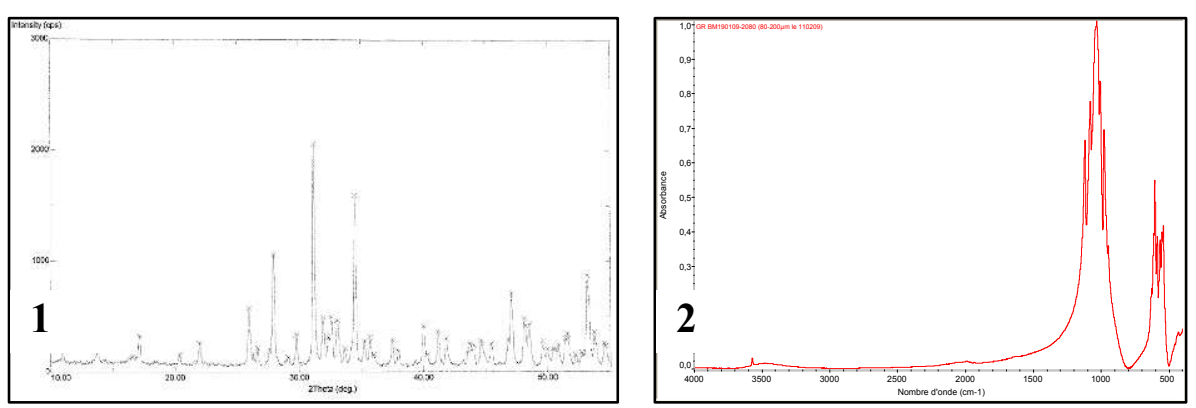
The crystal size and shape and micro and macropore distribution were determined by SEM (figure 3) Numerous concavities on the granules increased the developed surface for cell colonization. At the crystal level, homogeneous crystal size was observed with high amount of inter crystal spaces (micropores) fully interconnected (figure 4). The single crystals observed are not representative of individual crystal of HA or individual crystal of TCP, confirming the intimate mixture of HA/TCP in the synthesis of the MBCP+ smart scaffold.
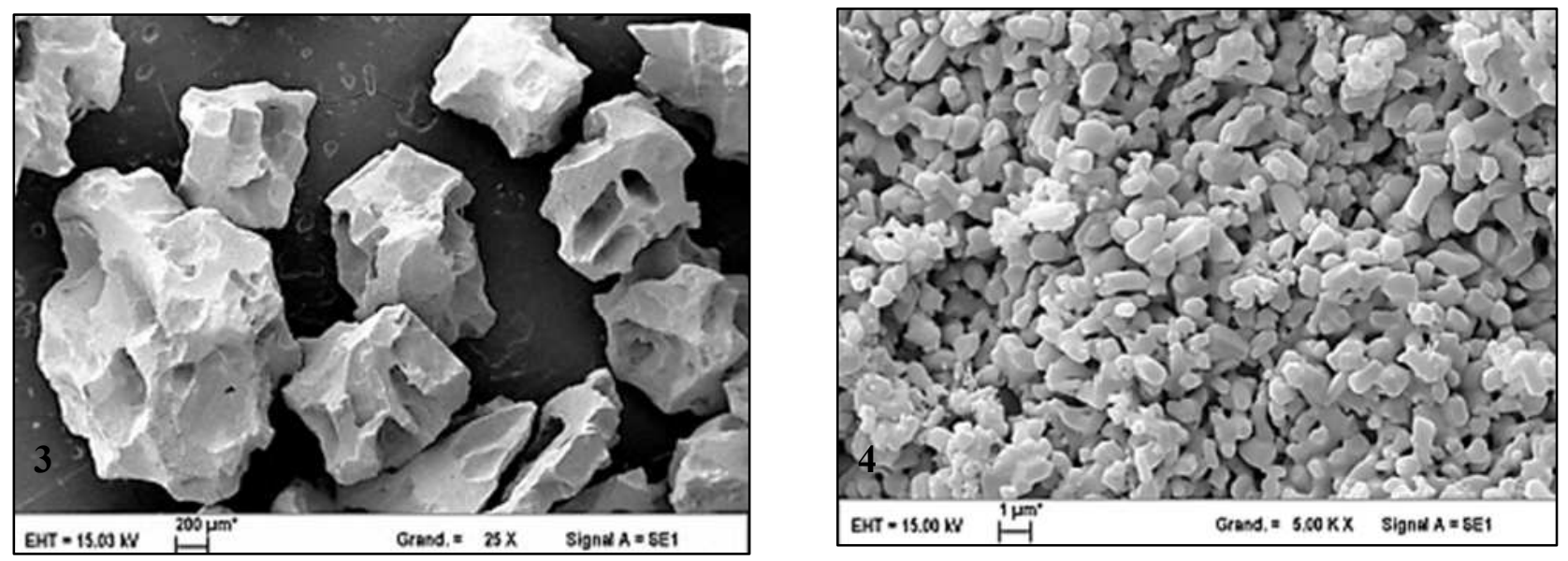

Figure 3: SEM, low magnification (x25) of granules of 0.2 to $0.5 \mathrm{~mm}$ showing numerous concavities Figure 4: SEM high magnification (x5000) showing single crystals of HA/TCP and the homogeneous micropores distribution

Using hrTEM of a single crystal of HA/TCP, we are unable to observe separate crystal of HA or TCP. The single crystal observed are a molecular mixture (nano crystallographic domains) constituted of the biphasic compound (figure 5). Convergent Beam Electron Diffraction had not enough resolution to characterize precisely the HA or $\beta$-TCP domain, the two crystallographic organization were intimately associated into the single crystals.

Figure 5: a hrTEM showing lattice place plane of HA/TCP. Numerous grain boundaries are observed between $B C P$ crystals (arrow). $b$, electron diffraction indicates molecular intimate mixture of HA and TCP.

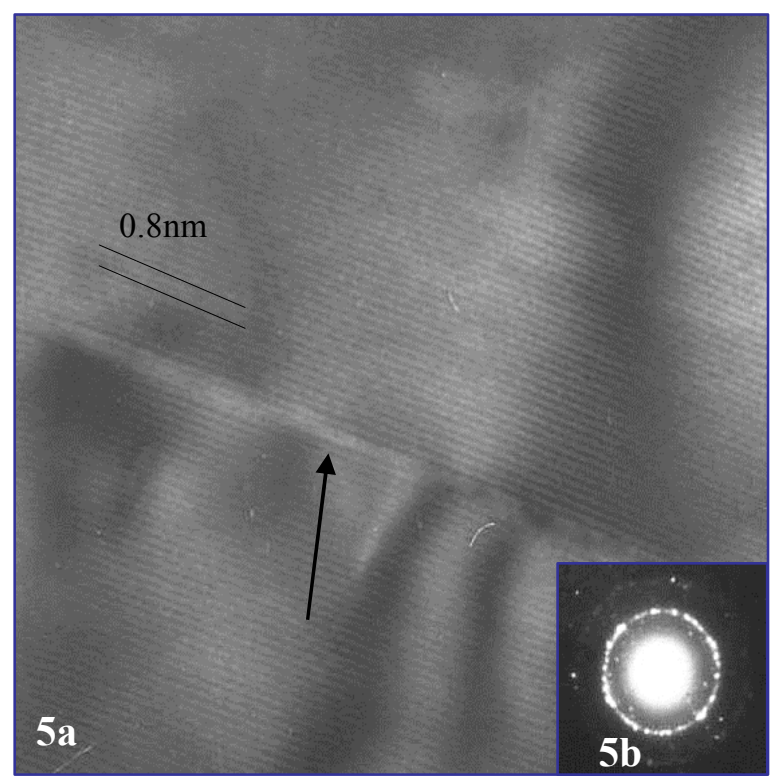

The crystal size is $0.5 \mu \mathrm{m}$ and the specific surface area was $6 \mathrm{~m}^{2} / \mathrm{g}$ for $\mathrm{MBCP}+$. Compression test showed $4 \mathrm{MPa}$. Mercury porosimetry are reported in table 1

Table 1: $M B C P+$ porosity distribution

\begin{tabular}{|l|l|}
\hline & MBCP+ \\
\hline Total porosity & $73 \%$ \\
\hline Macropores $>100 \mu \mathrm{m}$ & $19.6 \% \pm 2.4$ \\
\hline Mesopores 5 to $100 \mu \mathrm{m}$ & $39.8 \% \pm 3.1$ \\
\hline Micropores $<5 \mu \mathrm{m}$ & $40.6 \% \pm 3.2$ \\
\hline
\end{tabular}


The interconnections are evidenced by $3 \mathrm{D}$ reconstruction using qualitative and quantitative micro CT (figure 6).

\section{Figure 6: $3 D$ reconstruction of a block of $M B C P+$}

The incubation with bovine serum, indicated an increase of $30 \%$ of biological fluid absorption with the smart scaffold $\mathrm{MBCP}+$ compared to classical MBCP.

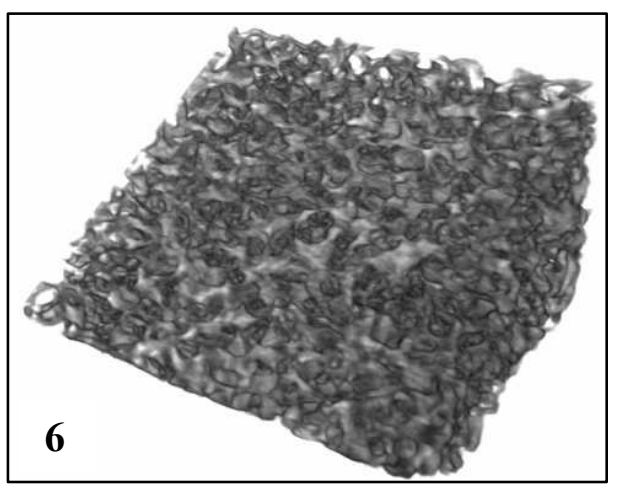

Faster bone ingrowth into the macropores was observed in short term implantation for MBCP+ comparing to classical MBCP. After 12 weeks no statistical difference was noticed between the 2 implants type. The rate of resorption however is higher for $\mathrm{MBCP}+: 17 \%$ versus $12 \%$ at 6 weeks, and $19 \%$ versus $17 \%$ after 12 weeks, (no significant difference) [5].

These kinetics of bone ingrowth at the expense of the $\mathrm{MBCP}+$ were confirmed in human clinical case (figure 7).

Figure 7: light microscopy, sinus lift after 4 months of implantation showing newly formed bone ( large arrow, pink) formed at the expense of the $M B C P+$ granules (*) particularly into the concavity of them (light arrow).

\section{Discussion}

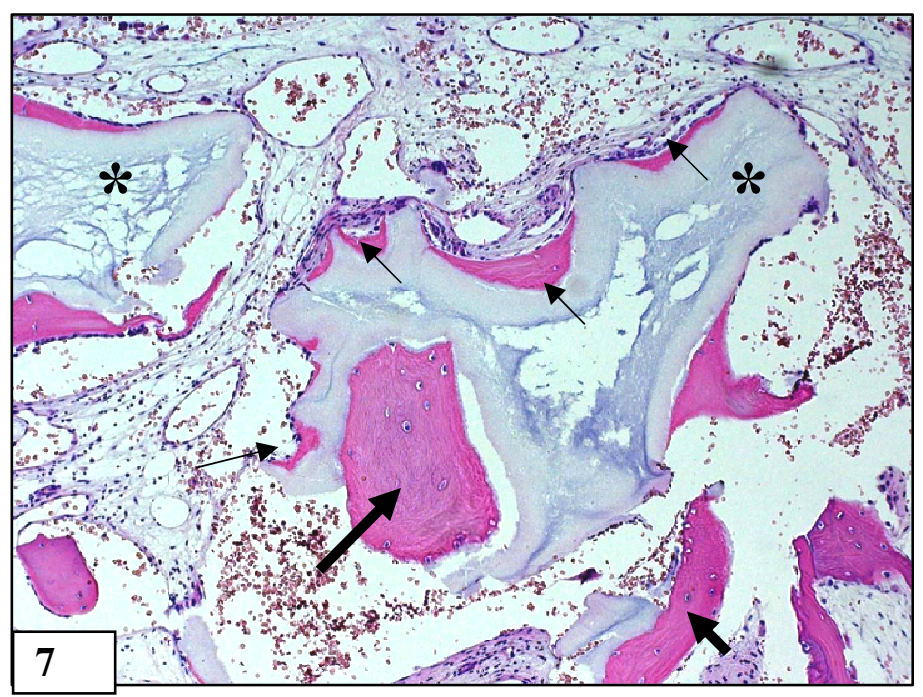

It has been shown recently that some bioceramics with particular micro structure induce bone formation after non bony site implantation in large animal models [8-11]. In bony site, the same materials have superior regenerative properties than other bioceramics or allogaft and autograft.

Apparently the low difference in total porosity (3\%) between the classical MBCP scaffold and smart scaffold as $\mathrm{MBCP}+$ types cannot explain higher permeability and osteogenic property. This higher permeability and absorption ability were essentially due to distribution of pore size particularly mesopores and the high micropore content representing around $40 \%$ of the total porosity. After implantation bone ingrowth is observed at the expense of the bioceramic, and newly-formed bone progressively replaced the bioactive material, followed by haversian bone remodelling [5].

The in vivo experiments indicated high cell colonization by osteogenic cells due to this interconnected and microporous structure associated to higher solubility. Moreover previous comparative study with equivalent macro and microstructure of different calcium phosphate bioceramics (HA, TCP or BCP 60/40 and BCP 20/80) demonstrates that the best scaffold was the ratio $20 / 80$ for tissue engineering i.e combination with stem cell cultivation and expansion, then implanted in non bony site [1]. Osteogenic or osteoinductive properties of calcium phosphate ceramics with an optimal micro and macro porosity have been also demonstrated without cell combination after implantation in non bony site (muscular area) [8,10,11]. Ripamonti has also postulated that the geometry of the material (concavity) is a critical parameter in bone induction [12]. The events and the origin of these important osteoinductive properties are the dissolution of the most soluble phase, the release of $\mathrm{Ca}$ and $\mathrm{P}$ ions, then the biological apatite precipitation concentrating the 
local growth factors [13]. The osteoprogenitor cells might in turn recognize the bone-like apatite layer formed in vivo by dissolution-reprecipitation on the material and produce mineralized bone.

The kinetic of bone ingrowth by the osteogenic cells differentiation needs to develop inside the macropores. Without macropores and mesopores, the bioactive processes are unable to develop in the deep of the implants. The association of dissolution at the crystal level, the diffusion of the biological fluid into the micropores, and the resorption by macrophages and osteoclastic cells of the materials at the surface and inside the macropores, involve a progressive bone substitution of the materials by physiological well vascularized bone. This is the common process of resorption-absorption/bone substitution of the Micro Macroporous Biphasic Calcium phosphate ceramics.

The intimate mixture of the process developed for smart scaffold $\mathrm{MBCP}+$ gave a unique and original property, contrarily to some others BCP described in the literature using a mechanical mixture of HA on one side and $\beta$-TCP on the other [14].

\section{Conclusion}

Advanced technologies for osteogenic smart scaffolds bioceramics for tissue engineering, involved higher efficacy of such matrices for further relevant surgical technologies as per surgery combination with bone marrow, or expanded stem cell in vitro for bone tissue engineering. The REBORNE project was able to demonstrate this strategy in maxillofacial and orthopaedic clinical situation.

The advanced $\mathrm{MBCP}+$ bioceramics technology involved higher efficacy of calcium phosphate scaffolds for further relevant surgical technologies fin bone tissue regeneration.

Acknowledgments: We thank the European Community 7 th framework program HEALTH-2009-1.4.2; on Regenerative Bone defects using New biomedical Engineering approaches (REBORNE, P. Layrolle coordinator).

We thank all the REBORNE partners for theirs contributions in the project.

\section{References}

[1] TL. Arinzeh, T. Tran, J. McAlary, G. Daculsi, Biomaterials. 26 (2005) 3631-3638.

[2] RF. Service, Tissue engineers build new bone, Science 289 (2000) 1498-1500.

[3] F. Jégoux et al., J Biomed Mater Res A 91(4) (2009) 1160-1169.

[4] F. Espitalier et al., Biomaterials 30 (2009) 763-769.

[5] G. Daculsi, F. Jegoux P. Layrolle, in Advanced Biomaterials: Fundamentals, Processing, and Applications book, Advanced Biomaterials: Fundamentals, Processing, and Applications, Bikramjit Basu, Dhirendra S. Katti, Ashok Kumar ed, John Wiley and sons Inc., 2009, pp.101-141.

[6] G. Daculsi et al., Key Engineering Materials. (2008) 361-363:1139-1142.

[7] G. Daculsi et al., J Mater Sci Mater Med 14(3) (2003) 195-200.

[8] G. Daculsi and P. Layrolle, Key Eng Mater 254 (2004) 1005.

[9] Yuan et al, Tissue Engineering (2006) 12:1607-15

[10] Habbibovic et al., Biomaterials 26 (2005) 3565.

[11] Le Nihouannen et al., Bone 36 (2005) 1086.

[12] U. Ripamonti, Biomaterials 17 (1996) 31.

[13] G. Daculsi et al., (1990), 46, 20-27.

[14] R. Oliveira Lomelino et al., J. Mater. Sci.: Mater Med 23 (2012) 781-788. 